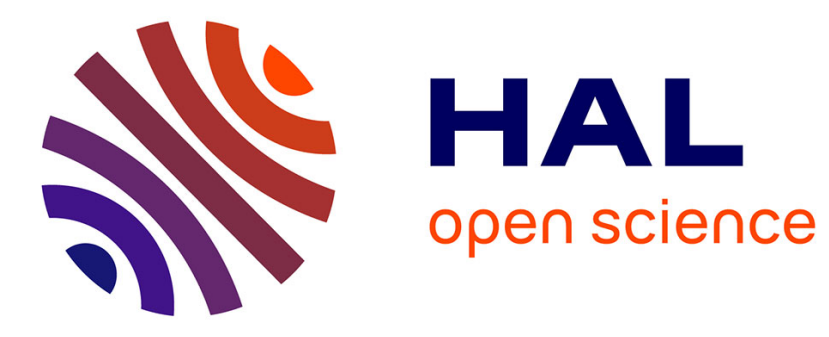

\title{
An example of in situ ductile damage analysis by tracking algorithm
}

Christophe Le Bourlot, Amin Azman, Jérôme Adrien, Éric Maire

\section{To cite this version:}

Christophe Le Bourlot, Amin Azman, Jérôme Adrien, Éric Maire. An example of in situ ductile damage analysis by tracking algorithm. Developments in X-Ray Tomography XII, Aug 2019, San Diego, United States. pp.40, 10.1117/12.2531357 . hal-02290954

\section{HAL Id: hal-02290954 https://hal.science/hal-02290954}

Submitted on 18 Sep 2019

HAL is a multi-disciplinary open access archive for the deposit and dissemination of scientific research documents, whether they are published or not. The documents may come from teaching and research institutions in France or abroad, or from public or private research centers.
L'archive ouverte pluridisciplinaire HAL, est destinée au dépôt et à la diffusion de documents scientifiques de niveau recherche, publiés ou non, émanant des établissements d'enseignement et de recherche français ou étrangers, des laboratoires publics ou privés. 


\title{
An example of in situ ductile damage analysis by tracking algorithm
}

\author{
Ch. Le Bourlot ${ }^{\mathrm{a}}$, A. Azman ${ }^{\mathrm{a}}$, J. Adrien ${ }^{\mathrm{a}}$, and E. Maire ${ }^{\mathrm{a}}$ \\ ${ }^{a}$ MATEIS, INSA Lyon, Villeurbanne, France
}

\begin{abstract}
Damage evolution in ductile metals is characterized by the nucleation, growth and coalescence of small internal voids. Many laws and studies have been developed to understand and predict damage. Numerical modeling, based on full field microstructure acquisition have proven also to be quite efficient. Real microstructure input are of utmost importance as some studies have shown that the deviation from the average behavior for voids growth can be huge and therefore allows a single cavity to drive the damage behavior and lead to failure. We develop the dedicated tool to analyze individually voids during in situ test and use results as input for numerical simulations.
\end{abstract}

Keywords: X-ray tomography, in situ, ductile damage, particle tracking

\section{INTRODUCTION}

Damage evolution in ductile metals is characterized by the nucleation, growth and coalescence of small internal voids. Many laws and studies have been developed to understand and predict damage. Numerical modeling, based on full field microstructure acquisitions have proven also to be quite efficient. ${ }^{1}$ Real microstructure input are of utmost importance as some studies have shown that the deviation from the average behavior for voids growth can be huge and therefore allows a single cavity to drive the damage behavior and lead to failure. ${ }^{2}$ In order to develop such approach, proper input data are needed. High energy X-ray contrast tomography allowed to work on large samples and avoid size and shape effect from samples.

The next step is to follow and correlate damage evolution during in situ traction test with numerical modeling. Voids nucleation and growth used to be characterized statistically as their number can be quickly very high $^{2,3}$ (few thousands on each tomographic volume acquisition). In such condition, the initial state is important but not enough to characterize the sample and be used as input. Following individually each cavity all along the mechanical test is of the utmost importance.

New generation synchrotrons allow fast acquisition and make it possible to easily collect few dozens of tomographic volume sets, often in situ with dedicated environments. ${ }^{3}$ But the analysis of such large amount of data requires then dedicated tools: an example is presented in this paper.

\section{DATASET ACQUISITION}

\subsection{In situ traction test experiment}

Interrupted in situ tensile tests have been performed on different materials (titanium, iron or aluminum alloys), to collect sets of tomographic volumes. The present paper focuses on the case of a titanium alloy, the T40. A home made tensile machine is used, ${ }^{4}$ mounted directly on the rotation stage.

Only large scale synchrotron facilities can allow simultaneously fast, efficient as well as high resolution tomography needed. High energy $(\approx 80 / 85 \mathrm{keV})$ is required to have a descent transmitted signal through thick samples. High brilliance is also needed to perform a single tomography acquisition in a short enough amount of time (few seconds to minutes) in order to minimize setup vibrations, sample relaxation,... Many volumes per

Further author information: (Send correspondence to Ch. Le Bourlot)

Ch. Le Bourlot: E-mail: christophe.le-bourlot@insa-lyon.fr 
samples (from 10 to 20) are acquired to properly describe the tensile curve. Acquisition have been acquired at SLS TOMCAT and Soleil PISCHE beamlines.

Fig. 1 shows a picture of the tensile rig, a sketch of the sample's geometry and a tensile curve of a T40. The samples are first machined as M3 screws, then a notch is machined to localized strain and therefor damages. The initial smallest diameter in the region of interest is $1 \mathrm{~mm}$, large enough to be sure to collect elementary representative volumes of each sample. Different radii are used for the notch in order to change the initial triaxality in the neck. ${ }^{5,6} \mathrm{~A}$ complete slice of the sample in the region of interest, where damage occurs, can be acquired with a lateral voxel size of $1 \mu \mathrm{m}$.
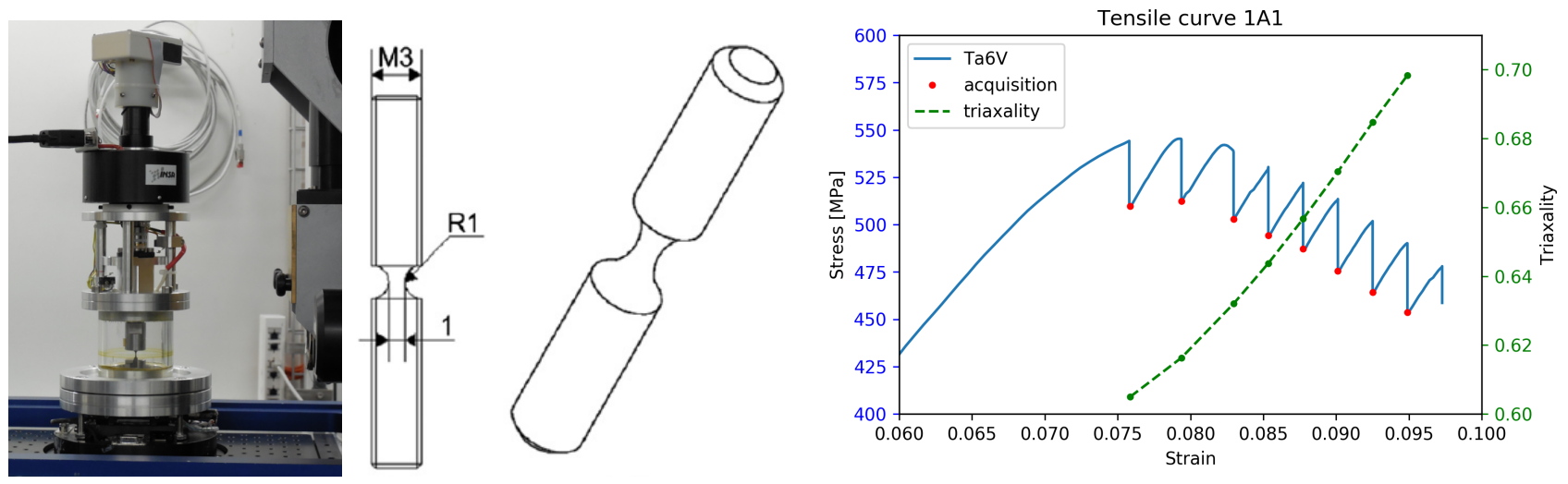

Figure 1. Left: picture of the in situ device mounted at TOMCAT beamline, as used in 4 ; Middle: shape of the sample with $R_{1}=1 \mathrm{~mm}$ as described in 2 ; right: strain/stress curve and associated triaxality calculated based on the acquired tomographic volumes.

An example of tensile curve is shown in Fig. 1 on the right. The tensile test is done with constant displacement of the upper sample grip, at $0.5 \mu \mathrm{m} / \mathrm{s}$. The displacement is stopped during the acquisition. The acquisition are done at the red dots, after relaxation and stabilization.

\subsection{Volume preparation and voids characterization}

The tomographic volumes are post-treated using the open source Fiji software. ${ }^{7}$ The volumes are 3D filtered before segmentation and binarization of the different phases. Each features are then labeled and series of characteristics are calculated, including: position, volume, shape, inertia, equivalent ellipsoid, surface,...


Figure 2. Left: voids segmented (4501) within the sample mask, for the last acquisition ; right: zoom at the center of the sample. 
First the surface of the sample is extracted. Potential open cracks at the surface are filled. From the evolution of the sample shape, the equivalent triaxality at the center of the sample is estimated (cf. Fig. 1 right).

The voids features are defined as void within the sample mask. Therefore a crack is counted as a void, and characterized as well.

The whole post-treatment process results in the creation of a database of all the voids in all the different acquisitions, with the associated parameters, and mesh-files with the sample shape and all the cavities shape. An example is shown on figure 2(left) with a zoom on the right.

\section{STATISTICAL CHARACTERIZATION}

A simple quantification of the damage can be done by analyzing this database.

\subsection{Nucleation, growth and germination estimation}

The distribution of the void size for each acquisition is presented in Figure 3. The number of voids per acquisition is given in the legend (in parenthesis). It is clearly visible that nucleation is very important, and that new nucleated voids are small. Void growth is visible by the shift of the voids distribution. Few very large voids would suggest coalescence events. When all the voids are taken into account, the average void size is almost constant: the growth of the void is compensated by the nucleation of new small voids. But the influence of these voids (the $n$ largest) on the ductile damage and especially to the finale failure is unknown.

The void growth behavior is then difficult to evaluate directly as all nucleation, growth and coalescence events happen simultaneously. A classical approach ${ }^{4,8,9}$ is to follow the $n$ largest voids assuming that the largest voids remain the same at each step, with different possible values for $n$. The result is shown on Figure 3(right). Whatever the number of voids taken into account, their average size increases during the experiment, confirming void growth.
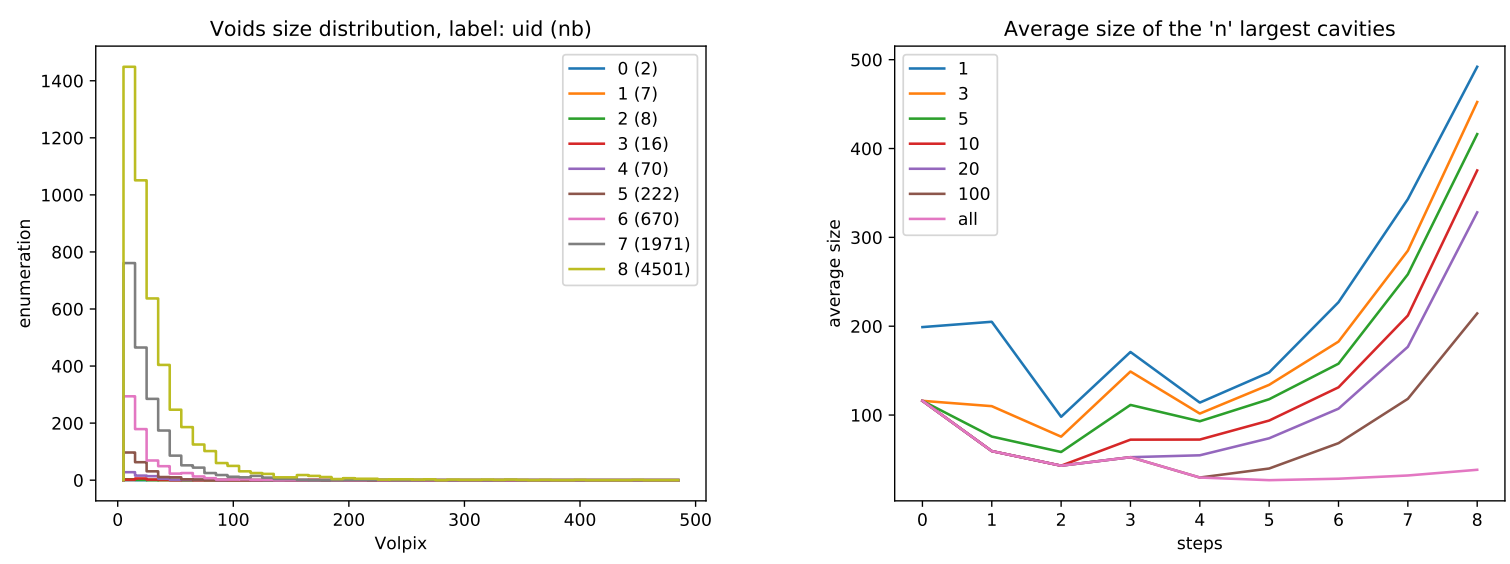

Figure 3. T40 sample voids statistics. left: voids distribution for the different acquisitions ; right: evolution of the average size of the $n$ largest voids for each acquisition. The fluctuation of the blue curve is due to segmentation uncertainties.

\subsection{Limitations}

Despite all the information collected, the characterization of the 3 stages of ductile damage (nucleation, growth and coalescence) is not clearly possible. For example, nucleation and coalescence have an inverse effect on the number of cavities and occur at the same time.

When acquisitions are limited to the central area of the sample, called local tomography, cavities may enter or exit the field of view during acquisition (the material moves during the tensile test).

Therefore, the only way to properly separate the different steps is to have a full knowledge of the behavior of each void ; which means being able to follow each of them. This can be achieved by using the tracking algorithm described below. 


\section{TRACKING ALGORITHM}

\subsection{Principle}

The theory of the tracking algorithm is classical (also named Particle Image Velocimetry ${ }^{10}$ ), and widely used in different fields: biology to follow cells evolution in situ using time-lapse videos, ${ }^{11-15}$ structural timber engineering, ${ }^{16}$ and much more...

The algorithm used here is an extension of previous publications, ${ }^{2,13}$ adapted to tomographic datasets.

The principle is to build a directed graph with each voids as nodes. The edges are computed between nodes with weights. The weight is the probability for the two nodes to be the same at consecutive time steps. Two more virtual nodes are added with which all nodes are connected: a black hole (representing the possibility for a track to get out of the field of view) and a white-fountain (this represent the possibility for a void to nucleate from nowhere).

Once build, all the voids tracks are extracted by a shortest path algorithm, using the black hole as an end target (by construction of the graph, all the tracks have to end in the black hole).

\subsection{Weight estimation}

Before any analysis, rigid body corrections are applied to each volume to correct possible movements during the tomographic acquisition (translations and rotations).

Simplest case The simplest case happens when all voids are perfect spherical voids during an in situ tensile test of a perfect elastic material. In that case, the macroscopic deformation of the sample is due to the tensile applied along one direction, and the Poisson effect for the two others. Geometrical corrections are simple.

Then the probability computed between two voids can be simply based on the distance between the geometrically corrected positions of the voids: voids would not move within the mater.

Real case As soon as the voids shape is no more spherical this approach is limited. For example, coalescence events produce highly non-spherical voids. To improve the weight estimation, few steps are added.

First, links between features should be computed only if the features are of same type (there could be no link between a void and a second phase inclusion for example).

Second, distance can be computed between surfaces of the voids rather than the center. As soon as superposition/intersection is detected between two voids of successive acquisition, their probabilities to be linked is very high (and the distance null). This computation used to be quite long, as it involves to load two meshes and compute distance between all their nodes.

If only ductile damage happens, the voids should grow only, they have no reason to shrink. Therefore if a link between two voids implies a volume diminution, this link should be remove (null probability).

Of course, these computations can be very quickly time consuming. To limit the memory usage and fasten the process, a cutting radius is defined: if the distance between two voids center is too high, the probability for these two to be linked is null, therefore there is no need to compute the distance between their surface.

\section{APPLICATION TO TITANIUM T40 SAMPLE}

The tracking algorithm is applied here to a T40 sample, deformed in situ. The figure 4 illustrates the result. Each line (or track) corresponds to the displacement of a cavity during tomographic acquisition. The cavities are connected to highlight this link. The color code is the acquisition step, from blue (first acquisition) to red (last acquisition). The vertical axis corresponds to the tensile direction clearly visible. The Poisson effect is also clearly visible (contraction along the two other directions).

The figure 5(left) illustrate the void growth on a single track due to damage: nucleation happens at acquisition step 3 and is followed during the next 5 steps. On the right is an example of a coalescence event: two cavities (in blue) coalesce giving the red one. The ligament between the two cavities is clearly visible (black arrow). 


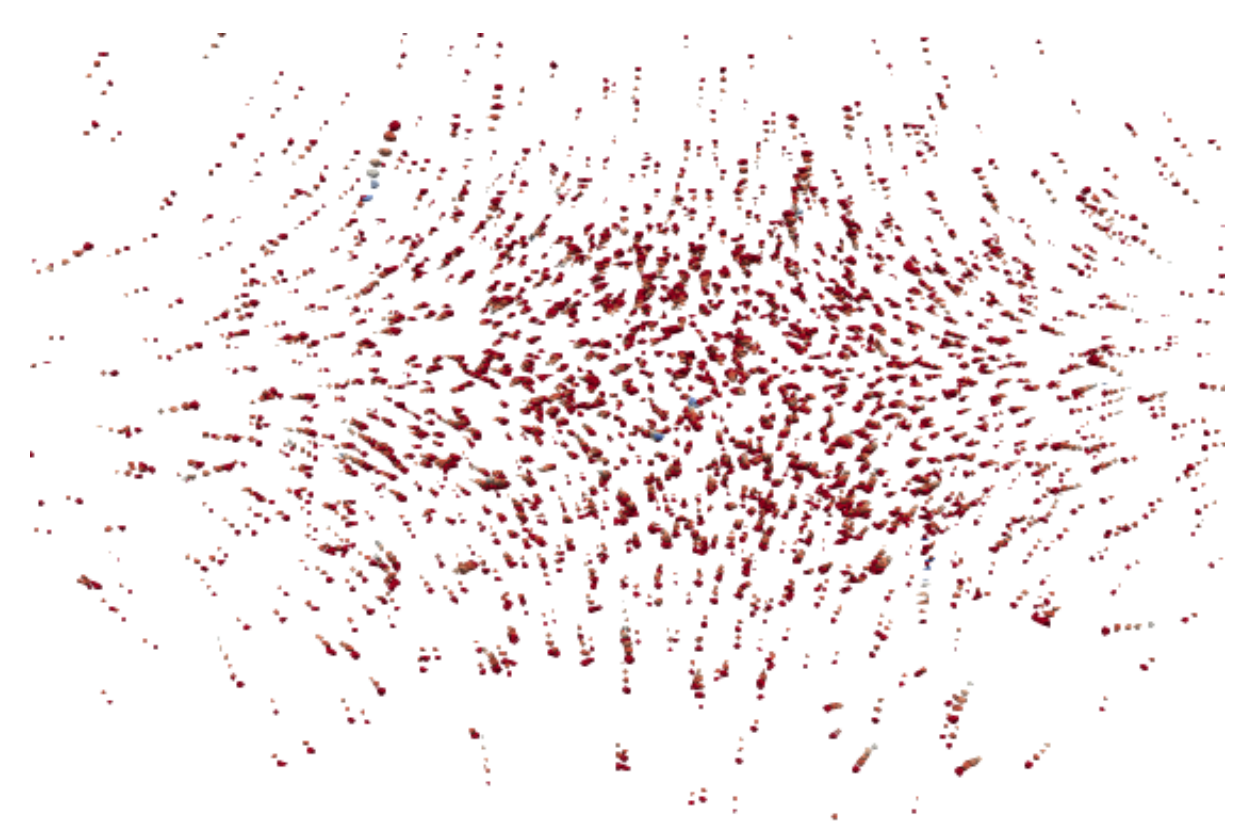

Figure 4. Tracks of the voids in the T40 sample. The color code is the acquisition step: from blue (first acquisition) to red (last one). The initial diameter is $1 \mathrm{~mm}$. The vertical axis corresponds to the direction of traction. The Poisson effect is clearly visible (extension along one axis, contraction along the other two).
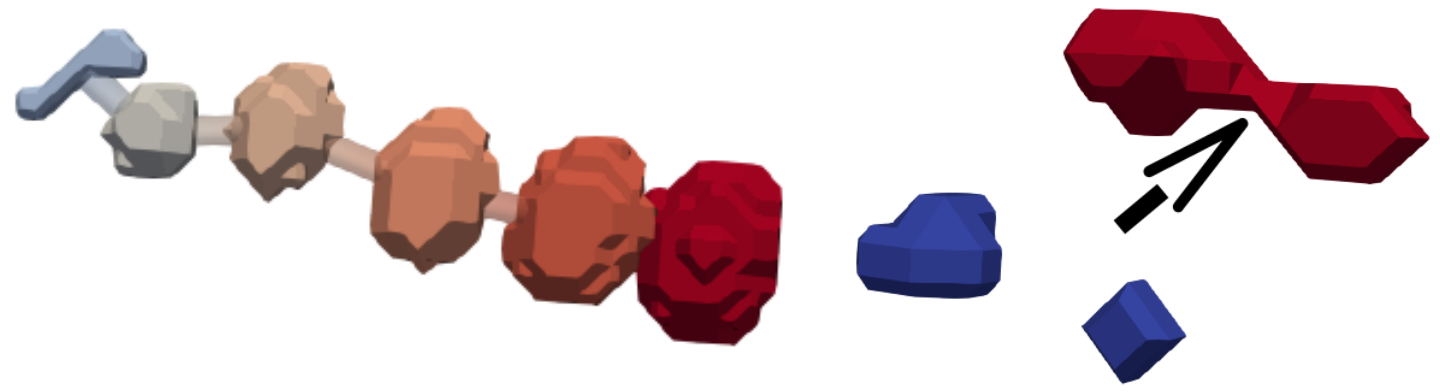

Figure 5. Left: example of a simple track, the color correspond to time step, the voids are linked to highlight the track. Right: the blue and red colors correspond to two successive acquisition time step. The black arrow point on the ligament.

Once the tracking done, nucleation and coalescence events are easy to separate. The Figures 6 illustrate: on the left the evolution of nucleation and coalescence events during the acquisition, and on the right the void growth.

As expected, nucleation is very important for this sample, but there is very few coalescence (about a dozen altogether). The interesting information comes from the voids growth evolution: some cavities are stable and do not show any growth evolution during deformation; on the other hand, others show a very high growth rate. This very large dispersion is independent of the initial volume of each cavity, or even its position in the sample. Therefore, the dispersion is in contradiction with the classical theory of void growth. This result has already been shown in 2, but this study generalizes the result: the dispersion of growth law was observed on all studied samples.

Once known, the evolution of the center of mass of each cavity and its surroundings provides access to the local deformation environment. Figure 7(left) illustrates the T40 sample using each cavity as a node of a Delaunay mesh generated using the Paraview application. ${ }^{17}$ The figure on the right shows the amplitude of the local equivalent elastic deformation brought back to a homogeneous and regular cubic mesh grid (voxels). The resolution of the resulting field depends on the cavity density (high in the center, low at the periphery of the 

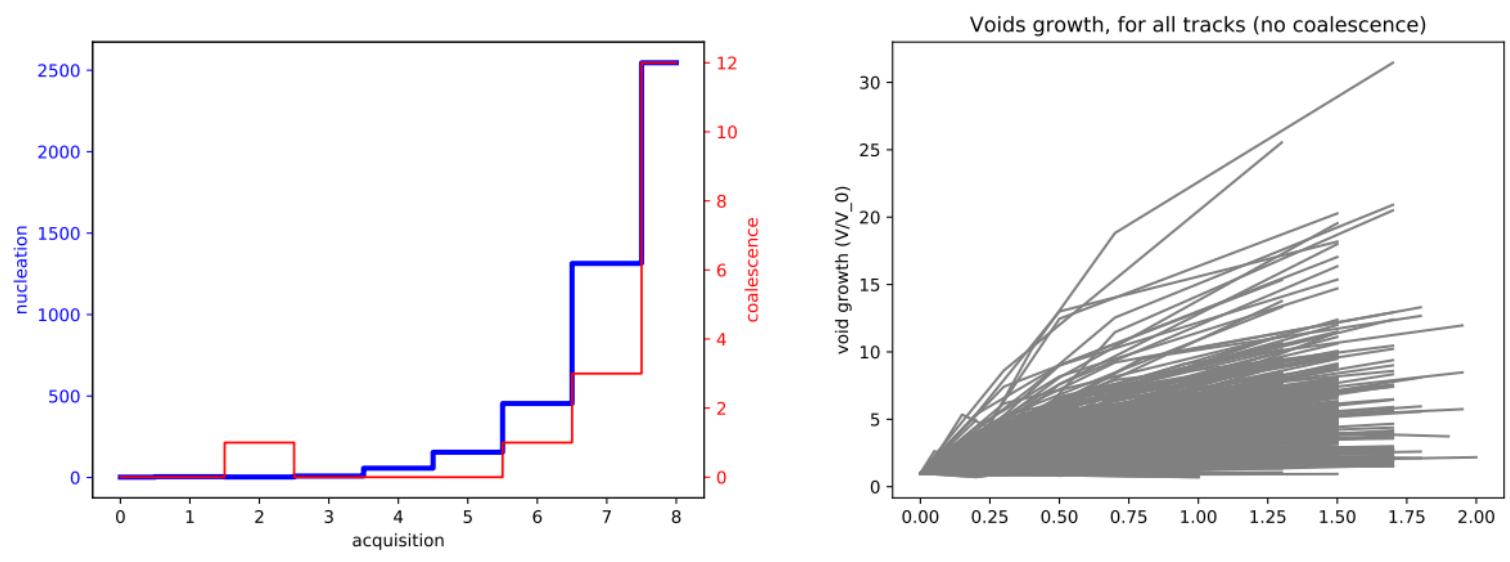

Figure 6. Left: evolution of nucleation and coalescence as function of the acquisition steps. Right: evolution of the volume of each cavity according to the effective deformation of the cavity (normalized with respect to the measured strain at nucleation).


Figure 7. Left: based on the tracked-voids position a mesh is generated using a Delaney tessellation ; Right: using finite element approach, the strain field associated to the mesh evolution/distortion between two acquisition is measured.

sample).

The local elastic deformation field can therefore be used as local information.

\section{ACKNOWLEDGMENTS}

The acquisitions have been done on TOMCAT beamline at SLS and PSICHE beamline at Soleil synchrotron facilities. Nothing would have been possible without the help of the beamline scientists.

\section{REFERENCES}

[1] Hannard, F., Pardoen, T., Maire, E., Le Bourlot, C., Mokso, R., and Simar, A., "Characterization and micromechanical modelling of microstructural heterogeneity effects on ductile fracture of $6 \mathrm{xxx}$ aluminium alloys," 103, 558-572 (2016).

[2] Lecarme, L., Maire, E., Kc, A. K., De Vleeschouwer, C., Jacques, L., Simar, A., and Pardoen, T., "Heterogenous void growth revealed by in situ 3-d x-ray microtomography using automatic cavity tracking," 63, 130-139 (2014).

[3] Maire, E. and Withers, P. J., "Quantitative x-ray tomography," 59(1), 1-43 (2014). 
[4] Maire, E., Le Bourlot, C., Adrien, J., Mortensen, A., and Mokso, R., "20 hz x-ray tomography during an in situ tensile test," 200(1), 3-12 (2016).

[5] Bridgman, P. W., [Studies in Large Plastic Flow and Fracture: With Special Emphasis on the Effects of Hydrostatic Pressure], Harvard University Press (1964). Google-Books-ID: MU5InwEACAAJ.

[6] Bai, Y., Teng, X., and Wierzbicki, T., "On the application of stress triaxiality formula for plane strain fracture testing," 131(2), 021002 (2009).

[7] Schindelin, J., Arganda-Carreras, I., Frise, E., Kaynig, V., Longair, M., Pietzsch, T., Preibisch, S., Rueden, C., Saalfeld, S., Schmid, B., Tinevez, J.-Y., White, D. J., Hartenstein, V., Eliceiri, K., Tomancak, P., and Cardona, A., "Fiji: an open-source platform for biological-image analysis," 9(7), 676-682 (2012).

[8] Lecarme, L., Tekog, C., and Pardoen, T., "Void growth and coalescence in ductile solids with stage III and stage IV strain hardening," 27(8), 1203-1223 (2011).

[9] Landron, C., Maire, E., Bouaziz, O., Adrien, J., Lecarme, L., and Bareggi, A., "Validation of void growth models using x-ray microtomography characterization of damage in dual phase steels," 59(20), 7564-7573 (2011).

[10] Adrian, R. J., "Twenty years of particle image velocimetry," 39(2), 159-169 (2005).

[11] Meijering, E., Dzyubachyk, O., Smal, I., and van Cappellen, W. A., "Tracking in cell and developmental biology," 20(8), 894-902 (2009).

[12] Chenouard, N., Smal, I., de Chaumont, F., Maka, M., Sbalzarini, I. F., Gong, Y., Cardinale, J., Carthel, C., Coraluppi, S., Winter, M., Cohen, A. R., Godinez, W. J., Rohr, K., Kalaidzidis, Y., Liang, L., Duncan, J., Shen, H., Xu, Y., Magnusson, K. E. G., Jaldn, J., Blau, H. M., Paul-Gilloteaux, P., Roudot, P., Kervrann, C., Waharte, F., Tinevez, J.-Y., Shorte, S. L., Willemse, J., Celler, K., van Wezel, G. P., Dan, H.-W., Tsai, Y.-S., de Solrzano, C. O., Olivo-Marin, J.-C., and Meijering, E., "Objective comparison of particle tracking methods," 11(3), 281-289 (2014).

[13] Brandes, S., Mokhtari, Z., Essig, F., Hnniger, K., Kurzai, O., and Figge, M. T., "Automated segmentation and tracking of non-rigid objects in time-lapse microscopy videos of polymorphonuclear neutrophils," 20(1), $34-51(2015)$.

[14] Ulman, V., Maka, M., Magnusson, K. E. G., Ronneberger, O., Haubold, C., Harder, N., Matula, P., Matula, P., Svoboda, D., Radojevic, M., Smal, I., Rohr, K., Jaldn, J., Blau, H. M., Dzyubachyk, O., Lelieveldt, B., Xiao, P., Li, Y., Cho, S.-Y., Dufour, A. C., Olivo-Marin, J.-C., Reyes-Aldasoro, C. C., Solis-Lemus, J. A., Bensch, R., Brox, T., Stegmaier, J., Mikut, R., Wolf, S., Hamprecht, F. A., Esteves, T., Quelhas, P., Demirel, ., Malmstrm, L., Jug, F., Tomancak, P., Meijering, E., Muoz-Barrutia, A., Kozubek, M., and Ortiz-de Solorzano, C., "An objective comparison of cell-tracking algorithms," 14(12), 1141-1152 (2017).

[15] Al-Zaben, N., Medyukhina, A., Dietrich, S., Marolda, A., Hnniger, K., Kurzai, O., and Figge, M. T., "Automated tracking of label-free cells with enhanced recognition of whole tracks," 9(1), 1-10 (2019).

[16] Ottenhaus, L.-M., Li, M., Nokes, R., Cammock, P., and McInnes, B., "Use of particle tracking velocimetry in timber material and connection testing," 77(2), 195-209 (2019).

[17] Ayachit, U., [The ParaView guide: updated for ParaView version 4.3], Kitware, full color version ed. (2015). OCLC: 944221263. 